This item was submitted to Loughborough's Research Repository by the author.

Items in Figshare are protected by copyright, with all rights reserved, unless otherwise indicated.

Time series count data models: an empirical application to traffic accidents

PLEASE CITE THE PUBLISHED VERSION

PUBLISHER

(C) Elsevier

VERSION

AM (Accepted Manuscript)

LICENCE

CC BY-NC-ND 4.0

REPOSITORY RECORD

Quddus, Mohammed A.. 2019. "Time Series Count Data Models: An Empirical Application to Traffic Accidents”. figshare. https://hdl.handle.net/2134/5308. 
This item was submitted to Loughborough's Institutional Repository (https://dspace.lboro.ac.uk/) by the author and is made available under the following Creative Commons Licence conditions.

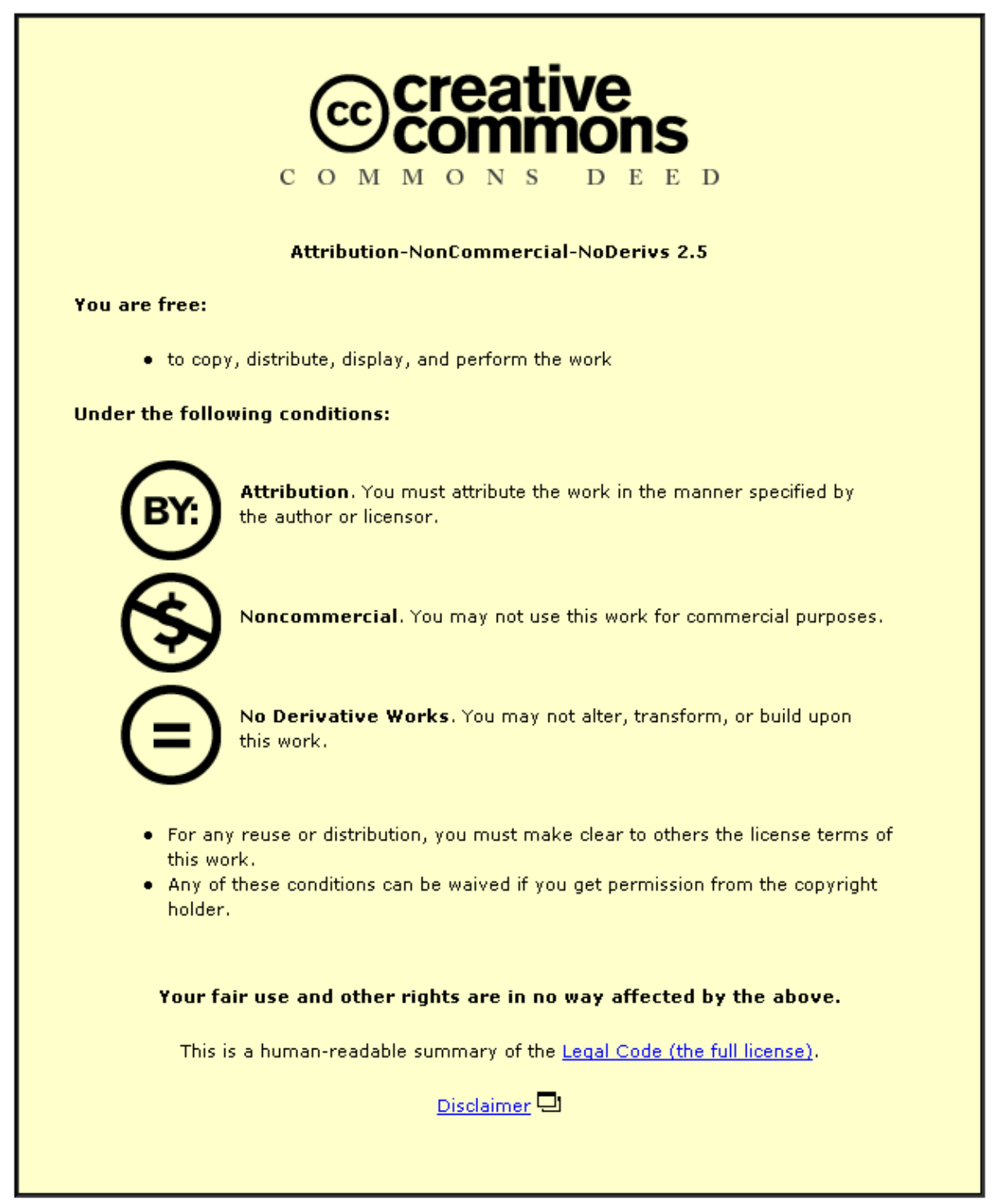

For the full text of this licence, please go to: http://creativecommons.org/licenses/by-nc-nd/2.5/ 


\title{
TIME SERIES COUNT DATA MODELS: AN EMPIRICAL APPLICATION TO TRAFFIC ACCIDENTS
}

\author{
Mohammed A Quddus \\ Transport Studies Group \\ Department of Civil and Building Engineering \\ Loughborough University \\ Leicestershire LE11 3TU \\ Tel: +44 (0) 1509228545 \\ Fx: + 44 (0) 1509223981 \\ Email: m.a.quddus@lboro.ac.uk
}

12 November 2007 


\begin{abstract}
:
Count data are primarily categorised as cross-sectional, time series, and panel. Over the past decade, Poisson and Negative Binomial (NB) models have been used widely to analyse cross-sectional and time series count data, and random effect and fixed effect Poisson and NB models have been used to analyse panel count data. However, recent literature suggests that although the underlying distributional assumptions of these models are appropriate for cross-sectional count data, they are not capable of taking into account the effect of serial correlation often found in pure time series count data. Real-valued time series models, such as the autoregressive integrated moving average (ARIMA) model, introduced by Box and Jenkins have been used in many applications over the last few decades. However, when modelling non-negative integer-valued data such as traffic accidents at a junction over time, Box and Jenkins models may be inappropriate. This is mainly due to the normality assumption of errors in the ARIMA model. Over the last few years, a new class of time series models known as integer-valued autoregressive (INAR) Poisson models, has been studied by many authors. This class of models is particularly applicable to the analysis of time series count data as these models hold the properties of Poisson regression and able to deal with serial correlation, and therefore offers an alternative to the real-valued time series models.
\end{abstract}

The primary objective of this paper is to introduce the class of integer-valued autoregressive (INAR) models for the time series analysis of traffic accidents in Great Britain. Different types of time series count data are considered: aggregated time series data where both the spatial and temporal units of observation are relatively large (e.g., Great Britain and years) and disaggregated time series data where both the spatial and temporal units are relatively small (e.g., congestion charging zone and months). The performance of the INAR models is compared with the class of Box and Jenkins realvalued models. The results suggest that the performance of these two classes of models is quite similar in terms of coefficient estimates and goodness of fit for the case of aggregated time series traffic accident data. This is because the mean of the counts is high in which case the normal approximations and the ARIMA model may be satisfactory. However, the performance of INAR Poisson models is found to be much better than that of the ARIMA model for the case of the disaggregated time series traffic accident data where the counts is relatively low. The paper ends with a discussion on the limitations of INAR models to deal with the seasonality and unobserved heterogeneity.

Keywords: Traffic accidents, Time series count data, Integer-valued autoregressive, Negative binomial, Accident prediction models 


\section{INTRODUCTION}

Road transport brings huge benefits to society, but it also has both direct and indirect costs. Direct costs include the costs of providing road transport services such as infrastructure, equipments, and personnel. Indirect costs include road transport accidents, travel delay due to road traffic congestion, and air pollution from road traffic. Among all of these costs, the cost associated with road traffic accidents is very high. According to the UK Department for Transport (DfT, 2003), the value of preventing a fatality (VPF) for the roads is $£ 1.25$ million (at 2002 price). Although UK is one of the safest countries in the world in terms of accident per veh-km travelled, the total number of fatalities from road traffic was 3,201 in 2005. One of the best ways to understand the causes of road traffic accidents is to develop various accident prediction models which are capable of identifying significant factors related to human, vehicle, socio-economic, road infrastructure, land-use, and the environment. For instance, Noland and Quddus (2004) developed an accident prediction model and reported that the improvements in medical technology and medical care reduced UK traffic-related fatalities. Based on the outcomes of accident prediction models, different countermeasures are implemented to reduce the frequency of road traffic accidents. Accident forecasting models are used to monitor the effectiveness of various road safety policies that have been introduced to minimise accident occurrences. For example, Houston and Richardson (2002) developed an accident-forecasting model and concluded that the change of an existing seat belt law from secondary to primary enforcement enhances road traffic safety. However, the performance and validity of these accident models largely depend on the selection of appropriate econometric models. In order to identify an appropriate econometric model, the understanding of different count variables is essential as road traffic accidents are non-negative, discrete, and sporadic event count.

Since road traffic accidents are non-negative, integer, and random event count, the distribution of such events follow a Poisson distribution. The methodologies to model accident counts are well developed. For instance, cross-sectional count data are modelled using a Poisson regression model (Kulmala, 1995). Since accident count data are normally over-dispersed (i.e., variance is greater than mean), a Negative Binomial (NB) regression model which is a Poisson-gamma mixture is more appropriate to apply (Abdel-Aty and Radwan, 2000; Lord, 2000, Ivan et al., 2000). If such cross-sectional count data contain many zero observations (i.e., excess zero-count data), then a zero-inflated Poisson (or NB) model or the Hurdle count data model is more appropriate ${ }^{1}$ (Land at al., 1996). If cross-sectional accident count data are truncated or censored, such as the number of fatalities per fatal accident in which the count data are truncated at one as there should be at least one fatality in a fatal accident,

\footnotetext{
${ }^{1}$ Readers are referred to Lord et al. (2005) for an interesting discussion on the suitability of such models in predicting traffic accidents
} 
these data are modelled using either a truncated Poisson or a truncated NB model. If cross-sectional count data are under-reported such as the occurrence of slight injury or property-damage accidents, then an under-reported Poisson model is used. If accident count data are panel data, fixed effects (FE) Poisson (or NB) model or random effects (RE) Poisson (or NB) model is used (Chin and Quddus, 2003). For clustered panel count data, the generalised estimating equations (GEE) technique is employed (Lord and Persaud, 2000).

However, there is a lack of suitable econometric models within the accident modelling literature to model time series accident count data. Normally, this type of accident data is modelled using a Poisson regression model or a NB regression model that has a prevailing assumption that observations should be independent to each other. This suggests that these models are more suitable for cross-sectional count data. Modelling time series count data using these models may result inefficient estimates of the parameters as time series data are normally serially correlated. One simple solution would be to introduce a time trend variable as an explanatory variable in the model to control for serial correlation. For example, Noland et al. (2006) used a NB model with a trend variable to study the effect of the London congestion charge on traffic casualties. However, there is no guarantee that this will explicitly account for the effect of serial correlation, specifically for the case of a long time series count data.

Time series models for continuous data are very well developed. Real-valued time series models, such as the autoregressive integrated moving average (ARIMA) model, introduced by Box and Jenkins (1970) have been used to model time series count data in many applications over the last few decades (e.g., Zimring 1975, Sharma and Khare, 1999, Houston and Richardson, 2002, Goh, 2005; Noland et al., 2006). However, when modelling non-negative integer-valued count data such as traffic accidents within a geographic entity over time, Box and Jenkins models may be inappropriate. This is mainly due to the normality assumption of errors in the ARIMA model. This largely suggests that a model is required which can take into account both the non-negative discrete property and autocorrelation of time series count data.

Over the last few years, a new class of such time series models known as integer-valued autoregressive (INAR) Poisson models, has been studied by many authors in the fields of finance, public health surveillance, travel and tourism, and forest sector etc. This class of models is particularly applicable to the analysis of time series count data as these models hold the properties of the distribution of count data and are able to deal with serial correlation, and therefore offers an alternative to the real-valued time series models and general Poisson or NB models.

The key objective of this paper is to introduce the class of INAR models for the time series analysis of accident count data from Great Britain. Two types of time series accident count data are considered: 
(1) aggregated time series data where both the spatial and temporal units of observation are relatively large (e.g., Great Britain and year), and (2) disaggregated time series data where both the spatial and temporal units of observation are relatively small (e.g., congestion charging zone in Central London and month). Various econometric models such as ARIMA, NB, NB with a time trend, and INAR(1) Poisson models are used to develop accident prediction models for each datasets. The performance of the INAR(1) Poisson model is compared with the other models.

The rest of the paper is organised as follows. The next section describes the class of INAR models used in this study. This is followed by a description of data sources used for the analysis. A presentation and interpretation of the results are then discussed in some detail. This paper ends with conclusions and limitations of this study.

\section{METHODOLOGY}

The model for continuous autoregressive pure time series data was introduced by Box and Jenkins (1970) and are now very well developed. The Box and Jenkins model such as the seasonal autoregressive integrated moving average (SARIMA) model is capable of taking into account the trend and seasonality (and hence the serial correlation) normally present in time series data. An extension of this model was proposed by (Box and Tiao, 1975) which has the ability to examine the effects of various regressors and interventions as explanatory variables along with the usual trend and seasonal components. This model can be expressed as follows (Hipel and McLEOD, 1994):

$$
y_{t}=\varpi_{0} I_{t}+\boldsymbol{\beta} \boldsymbol{X}+N_{t}
$$

in which $t$ is the discrete time (e.g., week, month, quarter, or year), $y_{t}$ is the appropriate Box-Cox transformation of $Y_{t}$, say $\ln Y_{t}, Y_{t}^{2}$, or $Y_{t}$ itself (Box and Cox, 1964), $Y_{t}$ is the dependent variable for a particular time $t, I_{t}$ is the intervention component, $\boldsymbol{X}$ is the deterministic effects of independent variables known as control variables $(X)$ and $N_{t}$ is the stochastic variation or noise component which can be represented by a ARIMA model denoted as ARIMA $(p, d, q)$ (for a non-seasonal time series) or a SARIMA model (for a seasonal time series) denoted as SARIMA $(p, d, q) \times(P, D, Q)_{S}$. In these models, $p$ is the order of the non-seasonal autoregressive (AR) process, $P$ is the order of the seasonal AR process, $d$ is the order of the non-seasonal difference, $D$ is the order of the seasonal difference, $q$ is the order of the non-seasonal moving average (MA) process, $Q$ is the order of the seasonal MA process, the subscript $s$ is the length of seasonality (for example $s=12$ with monthly time series data). The SARIMA model can be expressed as (Box et al., 1994): 


$$
N_{t}=\frac{\theta(B) \Theta(B) u_{t}}{\phi(B) \Phi\left(B^{s}\right)(1-B)^{d}\left(1-B^{s}\right)^{D}}
$$

in which $\phi$ and $\Phi$ are the regular and seasonal AR operators, $\theta$ and $\Theta$ are the regular and seasonal MA operators, $B$ and $B^{s}$ are the backward shift operators, and $u_{t}$ is an uncorrelated random error term with zero mean and constant variance $\left(\sigma^{2}\right)$. Details can be found in Box et al. (1994) for further explanation of this model.

The ARIMA- or SARIMA-based intervention model as shown in equation (1) is suitable for realvalued time series data as the error term is assumed to be normally distributed with zero mean and constant variance. Despite this assumption, this model is being used to investigate non-negative discrete time series processes related to a number of applications including road traffic accidents (e.g., Houston and Richardson, 2002; Noland et al., 2006).

There are a few major problems with the application of SARIMA models to non-negative integervalued time series process such as monthly accident count data. The first problem is the definition of the model. A real-valued autoregressive process of order 1 can be expressed as follows:

$$
Y_{t}=\alpha Y_{t-1}+e_{t}
$$

In order to obtain an integer valued $Y_{t}$ the following constraints have to be imposed on equation (3) such as (i) $e_{t}$ is integer valued and (ii) $\alpha=-1,0$, or 1 . Such constraints limit the practical use of realvalued autoregression time series process in the framework of count variables. The second problem concerns the commonly made assumption of normality. For a count variable in which the mean of the counts is relatively high such as yearly road traffic accidents in Great Britain, the distribution is usually found to be an approximate normal and hence, the use of SARIMA model may be satisfactory as the normality assumption is less questionable. However, for a count variable in which the mean of the count is close to zero such as monthly fatal road traffic accidents within a small geographic unit, the distribution is normally skewed to the right. Therefore, the assumption of normality, or of any other symmetric distribution, is unjustified.

The class of integer-valued autoregressive processes denoted by INAR have been studied by many authors (e.g., Al-Osh and Alzaid, 1987; McKenzie, E., 1988, Brännäs, Hellström, 2001, Karlis, 2006). A natural idea of such models is to replace the deterministic effect of lagged $Y_{t}^{\text {'s }}$ by a stochastic one 
(see equation 3). The approach developed replaces the scalar multiplication between $\alpha$ and $Y_{t-1}$ by binomial thinning which is defined as follows. If $Y_{t-1}$ is a non-negative integer and $\alpha \in[0,1]$ then

$$
\alpha \circ Y_{t-1} \equiv u_{1, t-1}+u_{2, t-1}+\ldots \ldots \ldots . .+u_{Y_{t-1}, t-1}=\sum_{i=1}^{Y_{t-1}} u_{i}
$$

where $\left\{u_{i}\right\}$ is a sequence of independently and identically distributed (IID) Bernoulli random variables, independent of $N$, and for which $\operatorname{Pr}\left(u_{i}=1\right)=1-\operatorname{Pr}\left(u_{i}=0\right)=\alpha$. It is noticeable that conditional on $Y_{t-1}, \quad \alpha \circ Y_{t-1}$ is a binomial random variable, the number of successes in $Y_{t-1}$ independent trials in each of which the probability of success is $\alpha$. Thus, the original real-valued AR(1) model of equation (3) is replaced by

$$
Y_{t}=\alpha \circ Y_{t-1}+e_{t}
$$

The thinning operation of $\alpha$ on $Y_{t-1}$ is independent of $e_{t}$. The second part of equation (5) consists of the elements which entered the system during the interval $[t-1, t]$ known as innovations. The basic derivation of the INAR process is based on the assumption that the innovations, $e_{t}$ has an independently and identically Poisson distribution i.e., $e_{t} \sim \operatorname{Poisson}\left(\lambda_{t}\right)$ where $\lambda_{t}$ is the Poisson mean denoted by

$$
\lambda_{t}=\exp \left(\beta X_{t}+\varpi_{0} I_{t}\right)
$$

The properties of the model in equation (5) can be found in Al-Osh and Alzaid (1987) and MaKenzie (1988). The mean and variance of the process $\left\{Y_{t}\right\}$ are equal to $\lambda /(1-\alpha)$. Equation (5) is termed as the Poisson INAR(1) which assumes that the underlying time series process is a stationary (Al-Osh and Alzaid, 1987; MaKenzie , 1988; Brännäs and Hall, 2001, Hellstrom, 2002).

Extensions of this model includes the Poisson INMA(1), the Poisson INARMA(1,1), the NB INAR(1) model, and the INARMA(1,1,) NB model. These are may be able to deal with both non-stationary and over-dispersed count data (Al-Osh and Alzaid, 1988; Brännäs and Hall, 2001, Karlis, 2006). Equation (5) can be estimated using the programmable Exact Maximum (EM) Likelihood algorithm (Karlis, 2006). Other models for time series of counts such as the serially correlated error model (Zegar, 1988) 
and the Zeger-Qaqish model (Zeger and Qaqish, 1988) can be found in Hellstrom (2002) and Kedem and Fokianos (2002).

\section{DATA}

Two datasets are used to investigate the appropriateness of different types of accident prediction models discussed above. One of these is a highly aggregated time series accident count and the other is a relatively disaggregated time series accident count.

The highly aggregated time series data considered in this study is the annual road traffic fatalities in GB between 1950 to 2005 obtained from the UK Department for Transport (DfT, 2006). The total number of observations is 55 and the mean and standard deviation of this time series process are 5,769 and 1,352 respectively. It is very well known that an accident model should contain an exposure to accident variable to control for total road traffic movements within the road network. The literature suggests that a good exposure to accident variable is vehicle kilometres travelled (VKT). The annual VKT data of GB are then collected from the DfT (DfT, 2006). Both annual road traffic fatalities and VKT data are shown in Figure 1. It is interesting to note that annual road traffic fatalities increase with the increase in VKT until 1966. Fatalities are then reduced with the increase in VKT. This is largely due to the implementation of different road safety measures, legislations, and policies over the years. For instance, the UK government introduced the seat-belt safety law in 1983 to reduce the severity of accidents. Penalty points for careless driving, driving with insurance, and seat- belt wearing for child passengers became law in 1989. The accident prediction model that will be developed using this dataset will also investigate the impact of these two interventions on road traffic fatalities while controlling for VKT.

\section{Figure 1 is about here}

The disaggregated time series data considered in this study is the monthly car casualties within the London congestion charging (CC) zone between January 1991 to October 2005 (Figure 2). This casualty data were obtained from the STATS19 database (1991-2004) and Transport for London (Jan 2005 - Oct 2005). The introduction of the congestion charge was postulated to reduce traffic casualties. According to Transport for London (TfL, 2006), there was an overall reduction of about 4070 casualty crashes a year during the charging hours within the charging zone. This is also noticeable from Figure 2 that the monthly car casualties reduce after the intervention. It is, therefore, our 
expectation that accident prediction models that will be developed in this study will discover this fact and will identify the impact of the introduction of the charge on car casualties. The total number of observations is 178 and the overall mean and variance of this time series process is 60.98 and 239.77. The total number of monthly road traffic accidents within greater London will be taken in all models as an exposure to risk of accidents for this dataset.

Figure 2 is about here

\section{RESULTS}

Different accident prediction models are developed using the econometric models such as ARIMA or SARIMA, NB, NB with a time trend, and INAR(1) Poisson models as described in section 2 for both aggregated and disaggregated time series datasets. Our main objective is to identify the best accident model for each type of time series datasets. For this purpose, each of the datasets is divided into two parts. One part is used to estimates the model parameters and the other part is used to validate the corresponding model using the estimated model parameters. The results for each of the datasets are presented below.

\section{Annual Road Traffic Fatalities in GB (Aggregated Time Series Process)}

The first part of the highly aggregated time series process representing the annual road traffic fatalities in GB contains observations from 1950 to 2000 resulting a total of 51 observations. This part of this time series process, usually known as a training dataset, are used to develop accident prediction models based on ARIMA, NB, NB with a trend, and INAR Poisson models. The rest of the observations (from 2001 to 2005) of this time series process, normally known as a validation dataset, is used to validate the developed accident prediction models. It is obvious from Figure 1 that this time series exhibits a downward trend suggesting that this dataset is non-stationary. This is also confirmed by the plot of the sample autocorrelation function (ACF) that clearly indicates serial correlation in the data as the autocorrelation coefficients at various lags fall outside the confidence limits (see Figure 3).

Form the plot of the road fatality series in Figure 1, it is not obvious that a data transformation is required. However, the variance appears to be decreasing from 1988 of this series. A Box-Cox transformation is applied with $\lambda=0$. Even after this transformation, there was a downward trend in the series which points out that non-seasonal differencing is needed for removing the non-stationary behaviour. After both transformation and differencing, the series looks stationary. However, it was difficult to determine the ARIMA model parameters using both ACF and partial ACF plots. The Box- 
Jenkins methodology is, therefore, employed to identify the most suitable ARIMA ${ }^{2}$ model based on the estimation sample. The values of $p, q$ are considered up to three and the final model is selected based on the Schwarz Information Criterion (SIC) with the requirement that all parameters are significant at the 95\% confidence level. The final model was $\operatorname{ARIMA}(1,1,1)$ suggesting that this stationary time series also has only a non-seasonal AR(1) and a non-seasonal MA(1) components.

\section{Figure 3 is about here}

It is worthwhile to note that the other models considered in this study such as NB, NB with a time trend, and INAR Poisson models assume that the underlying time series process is a stationary process and therefore, there is no need to manipulate the response variable of the process.

The results of ARIMA, NB, NB with a time trend variable, and INAR Poisson models are presented in Table 1. In each of these models, two interventions and one control variable are used as the explanatory variables and the annual road traffic fatalities in GB is used as a response variable. The first intervention variable is the introduction of the seat-belt law in 1983 and the second intervention variable is the introduction of various safety legislations in 1989. Both of these intervention variables are dummy variables represented by the so-called step functions. This suggests that these interventions cause an immediate and permanent effect on road traffic fatalities in GB. The control variable is the annual vehicle-kilometre travelled (VKT) in GB.

It can be seen that both intervention variables are statistically significant in all models except in the ARIMA (1,1,1) model. However, both AR1 and MA1 components of this ARIMA model are statistically significant at the $100 \%$ confidence level. The control variable, VKT, is also statistically significant in all models expect in the NB with a time trend model. This is due to the fact that the trend variable (linear) and the control variable (i.e., VKT) are highly correlated showing a correlation coefficient of 0.99 .

\section{Table 1 is about here}

The performance of each of the models presented in Table 1 can be found from the different "measures of accuracy" of the fitted models. These are the mean absolute percentage error (MAPE), the mean absolute deviation (MAD), the mean squared deviation (MSD), and the root mean squared error (RMSE). For all four measures, the smaller the value, the better the fit of the model. It can be seen that the best fitted model is the ARIMA(1,1,1) model in terms of all "measures of accuracy". The

\footnotetext{
${ }^{2}$ A SARIMA model is not applicable as this dataset is a non-seasonal time series
} 
performance of the INAR(1) Poisson model is also good relative to the ARIMA model. The worst performance model is found to be the NB model with a trend model for this dataset.

The validation dataset that contains observations from 2001 to 2005 is used to estimate the relative forecast error, $R F E$, (\%) of each models using the following equation:

$$
R F E=\sum_{i=1}^{5}\left(\frac{a b s\left(y_{i}-\hat{y}_{i}\right)}{y_{i}}\right) \times 100
$$

where, $y_{i}$ is the observed annual road traffic fatalities in GB and $\hat{y}_{i}$ is the forecasted annual road traffic fatalities using the developed model.

The results are shown in the last row of Table 1. The lowest RFE (2.79\%) is also found in the ARIMA $(1,1,1)$ model suggesting that the best performance model is the ARIMA $(1,1,1)$ model both in terms of the forecasted values associated with the out of sample observations.

In terms of the significant variables in the models, the two best performance models provide dissimilar results. Both intervention variables are found to be insignificant in the ARIMA model but found to be significant in all other models including the INAR(1) model. Both the seat-belt wearing law in 1983 and the different safety legislations in 1989 have a negative impact on road traffic fatalities in the UK in the INAR(1) model. This finding is consistent with the finding of other studies on seat-belt safety law (e.g., Houston and Richardson, 2002).

\section{Figure 4 is about here}

Figure 4 shows the graph of observed fatalities and predicted fatalities for the ARIMA, NB with a trend, and INAR(1) Poisson models from 1985 to 2005. It can be seen that the predicted fatalities of the ARIMA and INAR(1) Poison models are in-line with the observed fatalities for both within sample and out of sample observations. As expected, NB model with a time trend variable provides the worst fit.

\section{Monthly Car Casualties within the Congestion Charging Zone (Disaggregated Time Series Process)}

The training dataset for this time series process contains observations from January 1991 to December 2004 resulting a total of 168 observations over the 14 years. The validation dataset contains observations from January 2005 to October 2005. The plot of monthly car casualties versus time 
(Figure 2) reveals important characteristics about the observations. The sinusoidal curve (albeit relatively weak) in Figure 2 indicates that the data are seasonal. This is logical given that the exposure to accidents, the demand for travelling, is highest during the warmer summer months and lowest during the winter months. In addition, the decreasing trend component (from Oct 2002) indicates that the car casualty data in each month of the year are decreasing over time. This implies that monthly car casualty data within the congestion charging zone have both seasonal and trend components. This is also confirmed with a plot of the sample autocorrelation function (ACF) shown in Figure 5 which indicates that the series is non-stationary as some autocorrelation coefficients fall outside the $95 \%$ confidence limits. Firstly, a seasonal differencing is applied to the series and a plot of this differenced series exhibits a downward trend. This suggests that a non-seasonal differencing is also required (Hipel and McLEOD, 1994). The final series is presented in Figure 6 which implies that the series is a stationary process.

\section{Figure 5 is about here}

\section{Figure 6 is about here}

Both ACF and partial ACF plots of the final differenced series do not exhibit a pattern to identify a suitable SARIMA model. The Box-Jenkins methodology is employed to identify the most suitable SARMA model based on the estimation sample. The values of $p, P, q$ and $Q$ are considered up to three and the final model is selected based on the Schwarz Information Criterion (SIC) with the requirement that all parameters are significant (at the 95\% confidence level). The final model with the lowest SIC value was $\operatorname{SARMA}(0,1,1) \times(0,1,2)_{12}$ suggesting that this stationary time series also has only a nonseasonal MA(1) and two seasonal MA components such as SMA1 and SMA2.

The results of SARIMA, NB, NB with a time trend, and INAR(1) Poisson models are presented in Table 2. Each of these models has an intervention variable and a control variable. The intervention variable is the introduction of the London congestion charge in February 2003 which is assumed as a step function. The control variable is the total monthly road traffic accidents in greater London which is a direct measure of exposure to risk (Noland et al., 2006). It can be seen that the intervention variable, the introduction of the congestion charge, is statistically significant in all models. The coefficient value of this variable is found to be -0.31 in the INAR(1) model suggesting that the introduction of the congestion charging zone within central London reduces car casualties by about $27 \%$ if all other factors remain constant. The coefficient of the intervention variable in the SARIMA model suggests that there are 13 fewer fatalities (an average 33\% reduction) in each month after the introduction of the charge. The control variable is statistically significant in all models. Based on the various "Measures of Accuracy" and "Relative Forecast Error" of the developed models, it can be said 
that the best performance model is the INAR(1) Poisson model. The RFE calculated using equation (7) for the INAR(1) Poisson model is only $0.91 \%$. The worst performance model is the SARIMA model for which the $R F E$ is $1.36 \%$.

\section{Table 2 is about here}

In summary, it can be said that for the case of the aggregated time series count data the best accident prediction model is obtained when the real-valued ARIMA model is used and for the case of the disaggregated time series count data the best accident prediction model is achieved when the INAR(1) Poisson model is employed. It should be noted that both time series count datasets used in this study exhibits serial correlation and hence it is not surprising that none of the NB models (with a trend and without a trend) is found to be a suitable model for serially correlated time series count data as these models are unable to take into account the effects of serial correlation. This suggests that the integervalued discrete property of count data is not so important if the mean of the counts associated with a time series process are high. However, if the counts associated with a time series process exhibit low values, the distribution of count data follows a Poisson distribution and the properties of integervalued count data becomes important. This is confirmed by the results of the disaggregated time series data while the real-valued time series model provides the worst performance among all models. The INAR(1) Poisson model provides good results for both datasets.

\section{.5. CONCLUSIONS}

Accident prediction models for time series count data were developed employing a range of econometric models such as ARIMA, NB, NB with a time trend, and INAR(1) Poisson models. Two time series accident count datasets were used to develop the accident models in this study. One of the datasets was a highly aggregated time series process of annual road traffic fatalities in GB and the other dataset was a disaggregated time series process of monthly car casualties within the congestion charging zone. Both of the datasets had a problem of serial correlation. Each of these datasets was used to develop four accident prediction models based on the four econometric models while controlling for exposure to risk of accidents. The performance of the fitted models was investigated using various "Measures of Accuracy" for within sample observations and "Relative Forecast Error" for out of sample observations. The results implied that the best accident prediction model for the aggregated time series count data was achieved when the ARIMA model was used. The performance of INAR(1) Poisson model was also found to be good for this dataset. On the other hand, the best accident prediction model for the disaggregated time series count data was achieved when the INAR(1) Poisson model was used. This largely suggests that the controlling of serial correlation are important when the mean of the counts is relatively high. The preserving of integer structure of the 
count data is more important than the controlling of serial correlation if the mean of the counts is relatively low. Since INAR(1) Poisson model is capable of controlling both properties of time series count data and the ARIMA model is unable to pick up the effects of interventions (for the case of aggregated time series data), one should consider to employ an INAR model when developing accident prediction models for time series count data.

The INAR(1) Poisson process is a stationary time series process that has a limitation to deal with the presence of over-dispersion commonly found in accident data. The extensions of this model are an INAR(1) NB model or an INARMA(1,1) NB model that could potentially control for both nonstationary time series process and over-dispersion. However, the methods of estimating parameters for such models are very complex and are not readily available to the author to investigate in this study.

\section{ACKNOWLEDGEMENT}

The author would like to thank Dimitris Karlis from Athens University of Economics and Business for his invaluable help in estimating the INAR model.

\section{REFERENCES}

Abdel-Aty, M., Radwan E., 2000, Modeling Traffic Accident Occurrence and Involvement. Accident Analysis and Prevention 32(5), 633-642.

Al-Osh, M., Alzaid, A.A., 1987, First-order integer-valued autoregressive (INAR (1)) process. Journal of Time Series Analysis 8, 261-75.

Al-Osh, M., Alzaid, A.A., 1988, Integer-valued moving average (INMA) process. Statistical Papers 29, 281-300.

Alzaid, A. A., Al-Osh, M.,1990, An integer-valued pth-order autoregressive structure (INAR(p)) process. Journal of Applied Probability 27, 314-23.

Box, G. and Jenkins, G., 1970, Time series analysis: Forecasting and control, San Francisco: HoldenDay.

Box, G.E.P., Tiao, G.C., 1975, Intervention analysis with applications to economic and environmental problems. Journal of the American Statistical Association 70, 70-74.

Brännäs, K., Hall, A., 2001, Estimation in integer-valued moving average models. Applied Stochastic Models in Business and Industry 17, 277-91.

Brännäs, K., Hellström, J., 2001, Generalized integer-valued autoregression. Econometric Reviews 20, 425-43.

Chin, H.C., Quddus, M.A., 2003, Applying the random effect negative binomial model to examine traffic accident occurrence at signalized intersections, Accident Analysis \& Prevention, 35(2), 253259. 
DfT (Department for Transport), 2003, Highways Economics Note No1. 2002 - Valuation of the benefits of prevention of road accidents and casualties. Department for Transport, UK.

DfT (Department for Transport), 2006, Transport statistics Great Britain, $32^{\text {nd }}$ Edition, London: TSO.

Goh, B.H., 2005, The dynamic effects of the Asian financial crisis on construction demand and tender price levels in Singapore, Building and Environment, 40: 267-276.

Hellstrom, J., 2002. Count data modelling and tourism demand, Umea Economic Studies No. 584, Umea University, ISSN 0348-1018.

Hipel, K.W., McLEOD, A.I., 1994. Time series modelling of water resources and environmental systems, Elsevier, Amsterdam.

Houston, D.J., Richardson, L.E., 2002, Traffic safety and the switch to a primary seat belt law: the California experience, Accident Analysis and Prevention 34 (6): 743-751.

Ivan, J.N., Wang, C., Bernardo, N.R., 2000. Explaining two-lane highway crash rates using land use and hourly exposure. Accident Analysis Prevention 32 (6), 787-795.

Karlis, D., 2006, Time series model for count data, Paper presented at the Annual Conference of the Transportation Research Board, Wahsington, D.C.

Kedem, B., Fokianos, K., 2002. Regression models for time series analysis, John Wiley \& Sons, Inc., New Jersey.

Kulmala, R., 1995, Safety at Rural Three-and Four-arm Junctions: Development and Application of Accident Prediction Models. VTT publications. Espoo: Technical Research Center at Finland.

Land, K.C., McCall, P.L., Nagin, D.S., 1996, A Comparison of Poisson, Negative Binomial and Semi-parametric Mixed Poisson Regressive Models with Empirical Applications to Criminal Careers Data. Sociological Methods and Research, 24, 387-442.

Lord, D., 2000. The prediction of accidents on digital networks: characteristics and issues related to the application of accident prediction models. Ph.D. Dissertation, Department of Civil Engineering, University of Toronto, Toronto.

Lord, D., Persaud, B.N., 2000, Accident prediction models with and without trend: application of the generalized estimating equations procedure. Transportation Research Record 1717, 102-108.

McKenzie, E. , 1988, Some ARMA models for dependent sequences of Poisson counts. Advances in Applied Probability 20, 822-35.

Noland, R. B., Quddus, M.A, 2004, Improvements in Medical Care and Technology and Reductions in Traffic-related Fatalities in Great Britain, Accident Analysis and Prevention, 36(1): 103-113.

Noland, R.B., Quddus, M.A. and Ochieng, W.Y., 2006, The effect of the congestion charge on traffic casualties in London: an intervention analysis, Presented at the Transportation Research Board (TRB) Annual Meeting, Washington, D.C., USA, January.

Sharma, P., Khare, M., 1999, Application of intervention analysis for assessing the effectiveness of CO pollution control legislation in India, Transportation Research Part D 4: 427-432.

Transport for London (TfL), 2006. Central London Congestion Charging: Impacts Monitoring, Fourth Annual Report. Available on the internet at: http://www.tfl.gov.uk. Accessed May 2007 
Zeger, S.L., 1988. A regression model for time series counts, Biometrika, 75, 621-629.

Zegar, S.L., Qaqish, B. 1988. Markov regression models for time series: a quasi-likelihood approach, Biometrics, 44, 1019-1031.

Zimring, F., 1975, Firearms and federal law: the Gun Control Act of 1968. Journal of Legal Studies 4, 2 (January): 133-198. 


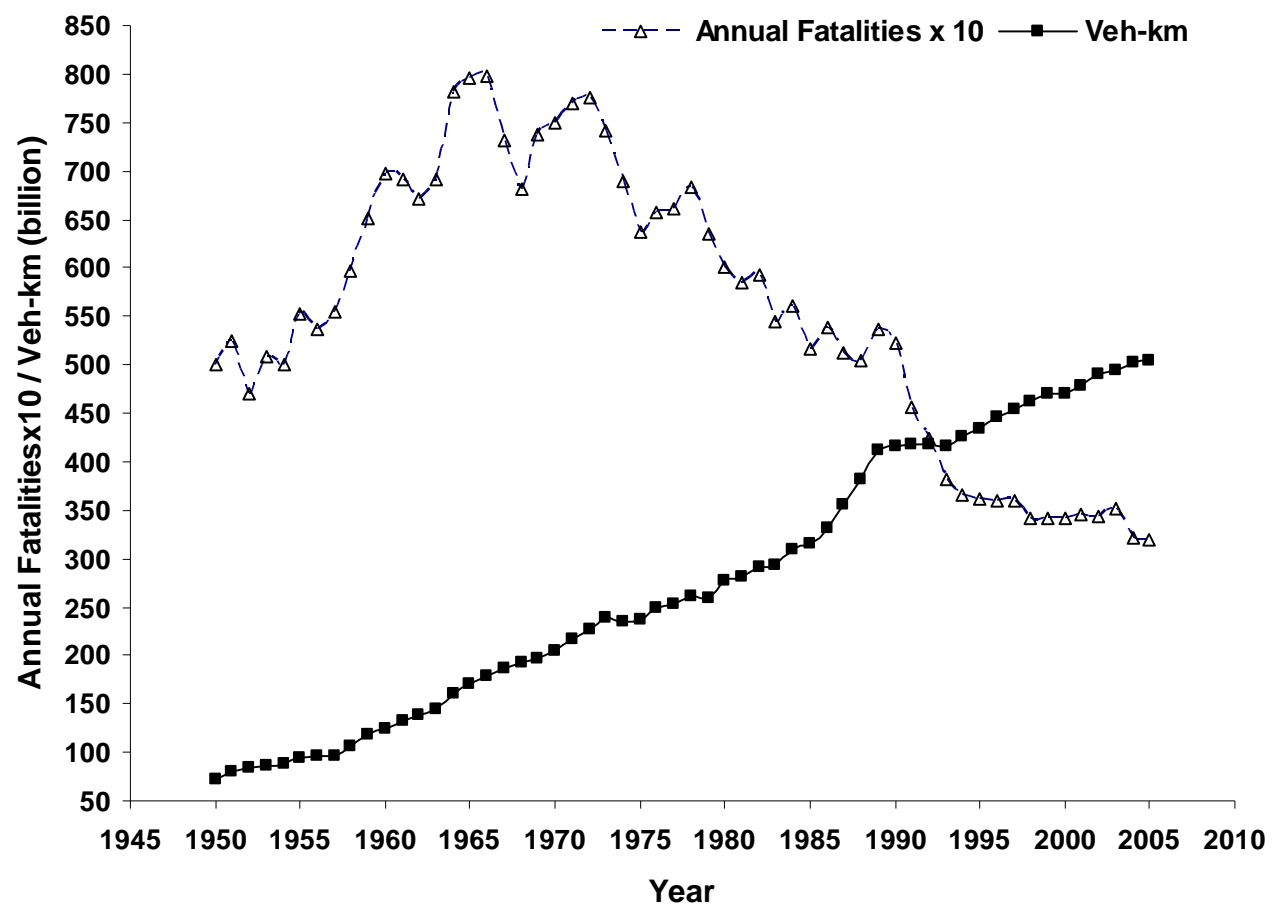

Figure 1: Annual road traffic fatalities and vehicle $\mathrm{km}$ travelled in GB 


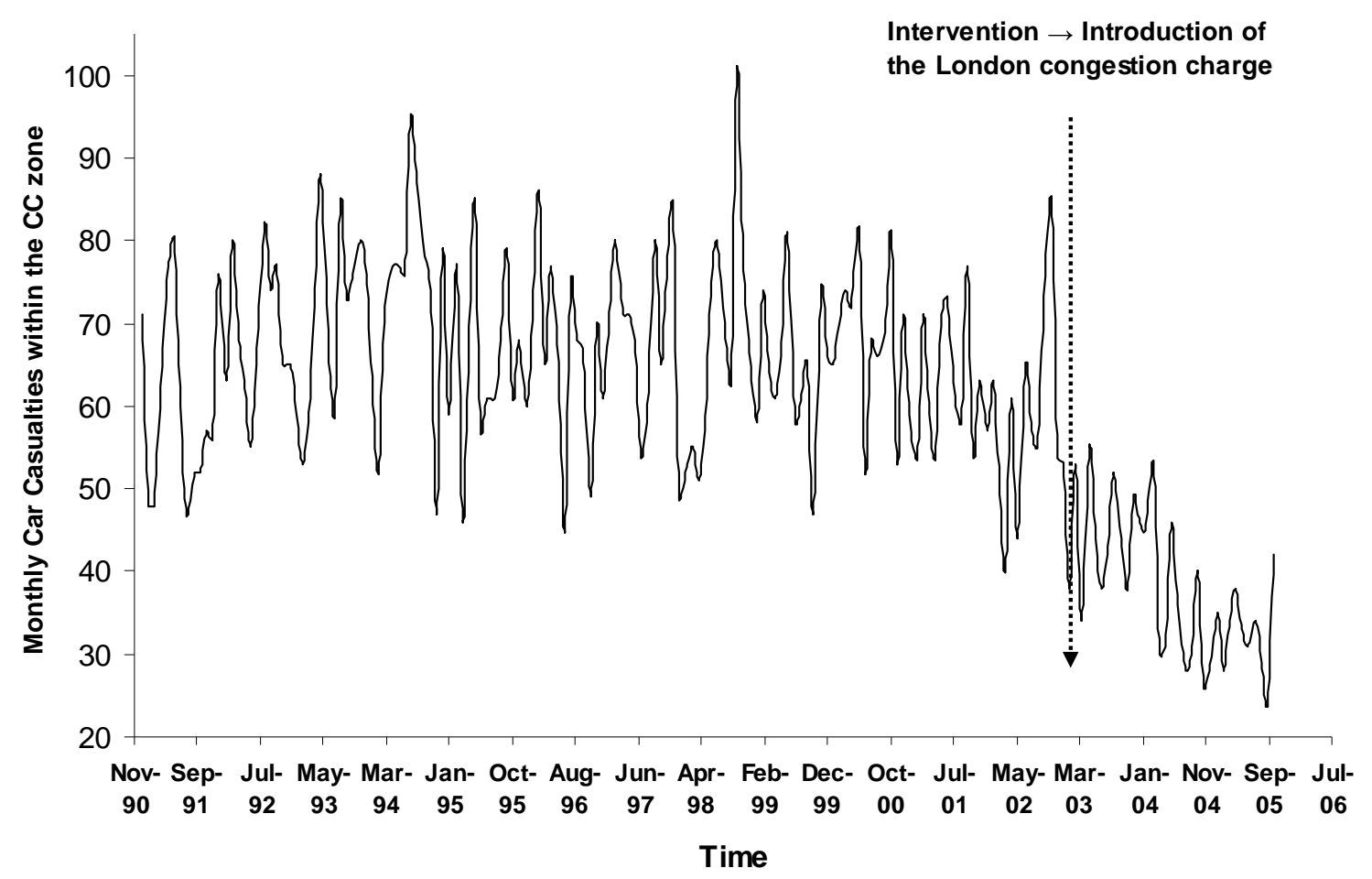

Figure 2: Monthly car casualties within the congestion charging zone (Jan 1991 to Oct 2005) 


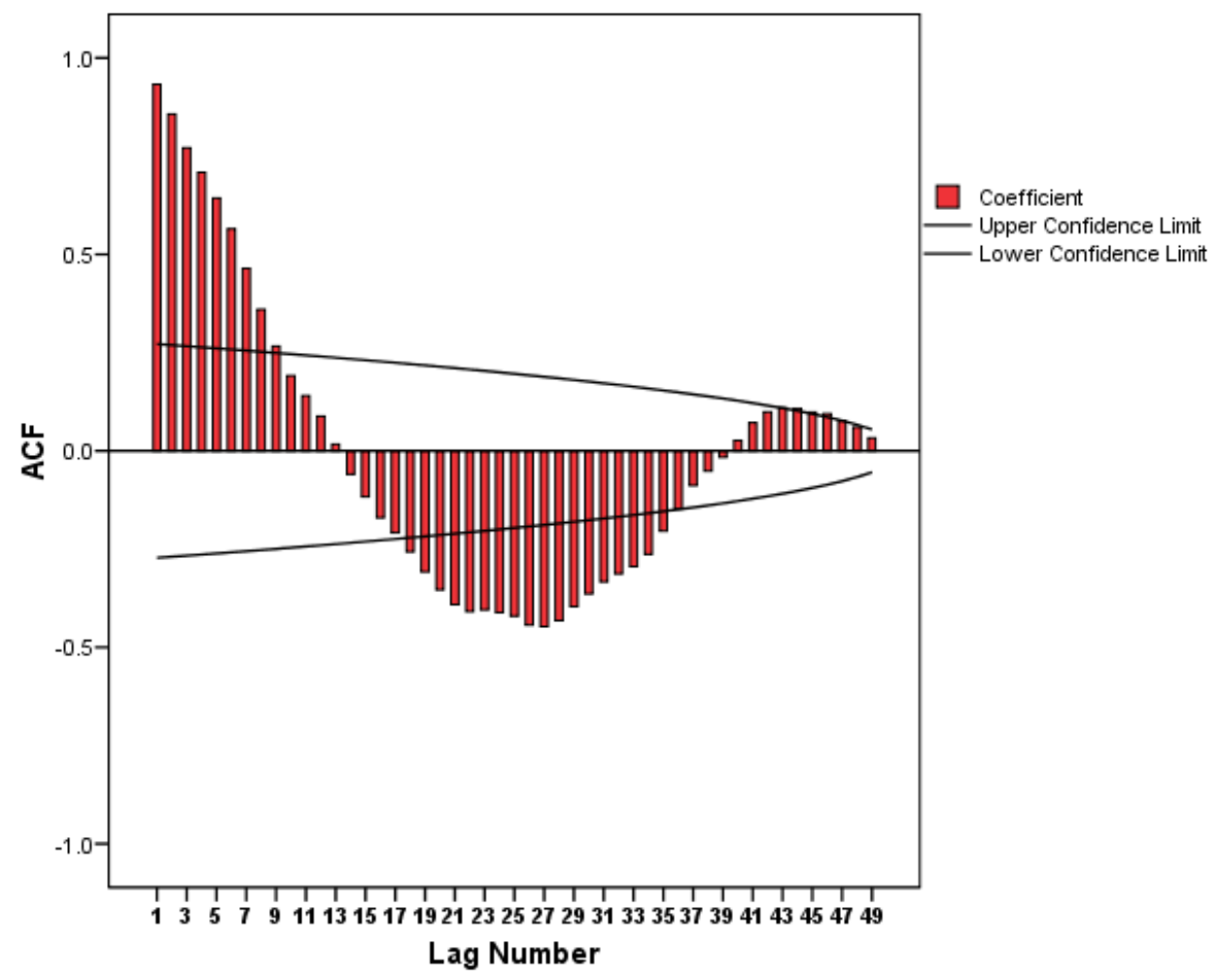

Figure 3: Sample ACF and 95\% confidence limits for the yearly road fatalities in GB 


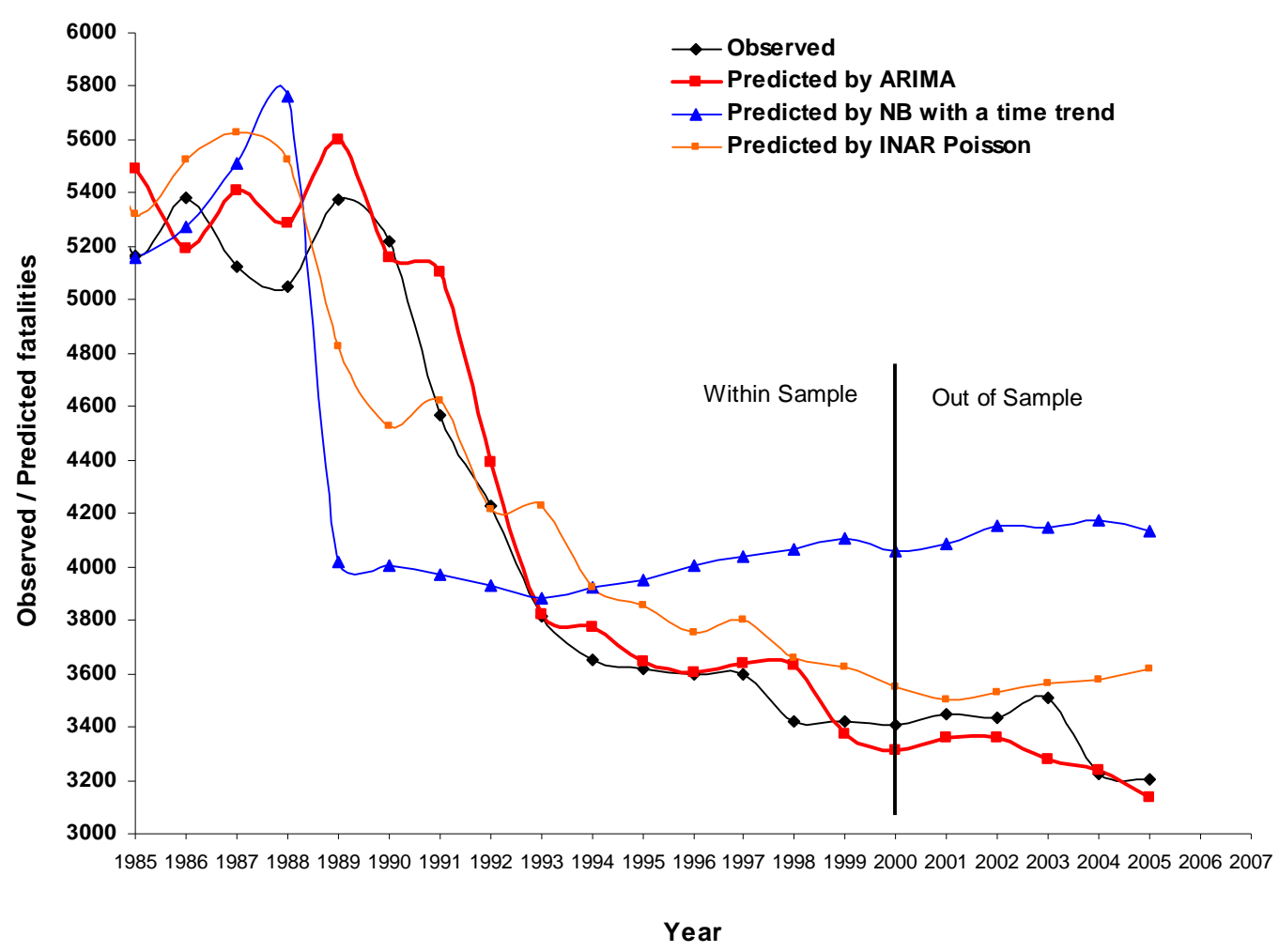

Figure 4: Observed vs Predicted values of fatalities 


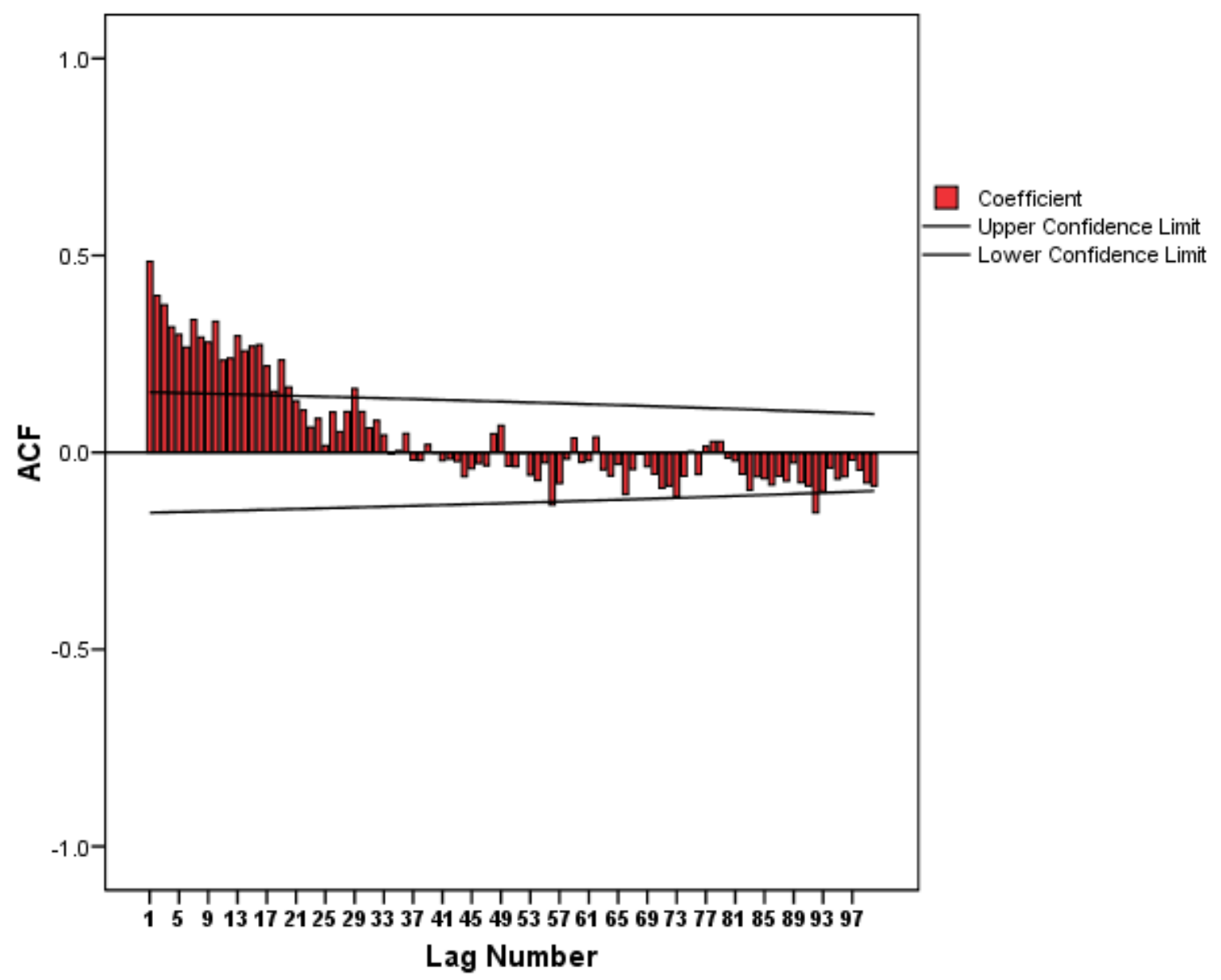

Figure 5: Sample ACF and 95\% confidence limits for the monthly car casualties within the congestion charging zone (Jan 1991 to Dec 2004) 


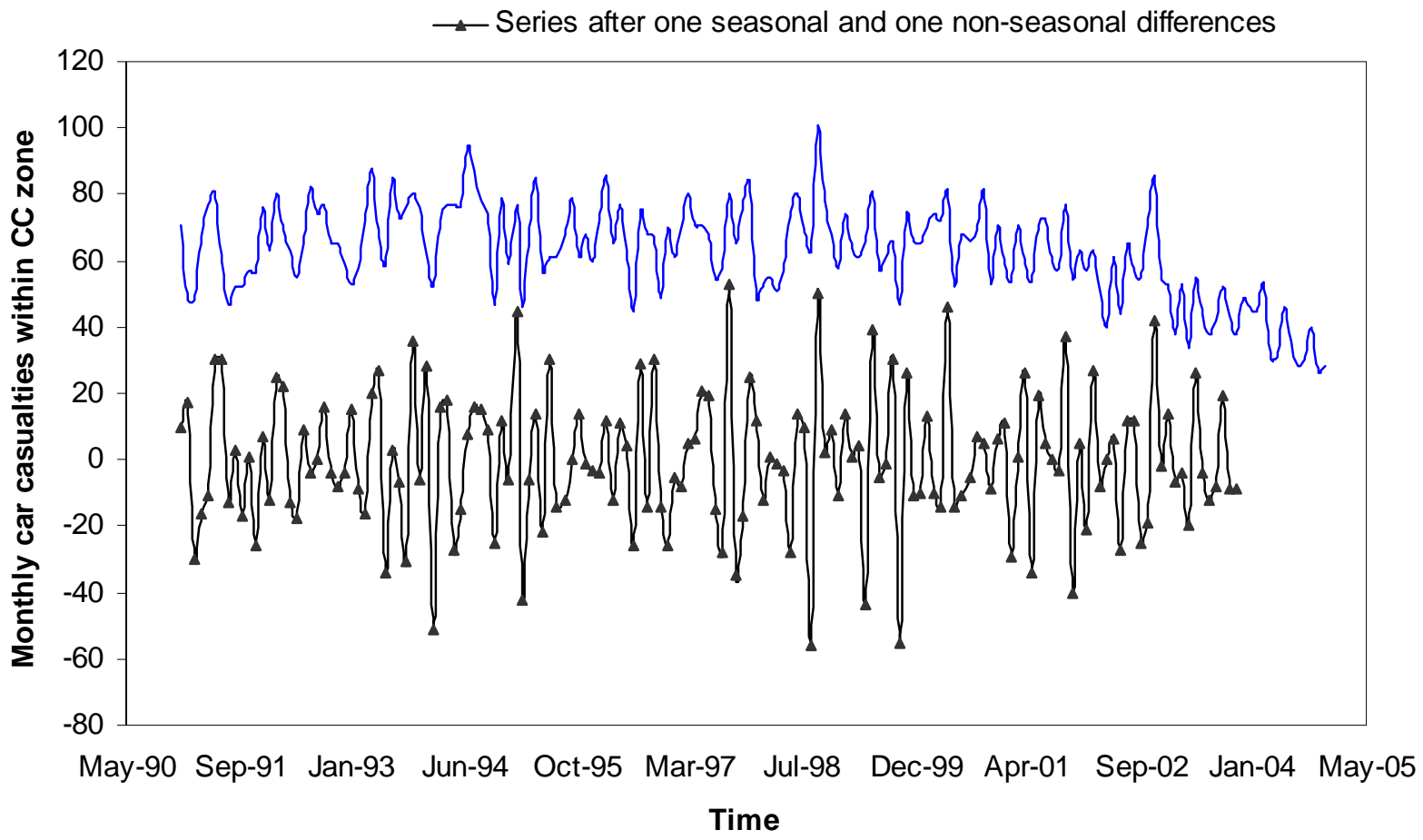

Figure 6: The differenced (seasonally and non-seasonally) monthly car casualty series for the congestion charging zone, from January 1991 to December 2004. 
Table 1: Accident prediction models for annual road traffic fatalities in GB

\begin{tabular}{|c|c|c|c|c|c|c|c|c|}
\hline \multicolumn{9}{|c|}{ Aggregate Time Series Accident Count Data (Yearly road fatalities in Great Britain 1950 - 2000) } \\
\hline & \multicolumn{2}{|c|}{ ARIMA $(1,1,1)$} & \multicolumn{2}{|l|}{ NB } & \multicolumn{2}{|c|}{ NB with a time trend } & \multicolumn{2}{|c|}{ INAR(1) Poisson } \\
\hline Explanatory Variables & Coeff & t-stat & Coeff & t-stat & Coeff & t-stat & Coeff & t-stat \\
\hline Seat-belt wearing law & -0.0449 & -0.84 & -0.3176 & -3.94 & -0.3336 & -4.00 & -0.3942 & -3.65 \\
\hline New legislation on safety & 0.0273 & 0.46 & -0.3588 & -4.65 & -0.4186 & -3.57 & -0.4236 & -2.95 \\
\hline Veh-km (billion) & 0.0031 & 2.48 & 0.0007 & 2.12 & 0.0022 & 1.01 & 0.0023 & 1.89 \\
\hline Trend (Linear) & - & - & - & - & -0.0107 & -0.68 & - & - \\
\hline Constant & - & - & 8.6481 & 131.14 & 8.5765 & -0.68 & 8.5157 & -1.44 \\
\hline Non-seasonal AR1 & 0.9736 & 14.80 & - & - & - & 68.97 & - & - \\
\hline Non-seasonal MA1 & 0.8251 & 4.97 & - & - & - & - & - & - \\
\hline \multicolumn{9}{|l|}{ Descriptive statistics } \\
\hline Overdispersion parameter & - & - & 0.0183 & 5.01 & 0.0181 & 5.01 & - & - \\
\hline Thinning parameter & - & - & - & - & - & - & 0.1250 & 3.02 \\
\hline Series of length & \multicolumn{2}{|c|}{51.00} & \multicolumn{2}{|l|}{51} & \multicolumn{2}{|c|}{51} & \multicolumn{2}{|c|}{51} \\
\hline Number of residuals & \multicolumn{2}{|c|}{50.00} & \multicolumn{2}{|l|}{51} & \multicolumn{2}{|c|}{51} & \multicolumn{2}{|c|}{51} \\
\hline Log-likelihood & \multicolumn{2}{|c|}{76.59} & \multicolumn{2}{|c|}{-410.94} & \multicolumn{2}{|c|}{-410.71} & \multicolumn{2}{|c|}{-406.21} \\
\hline \multicolumn{9}{|l|}{$\begin{array}{l}\text { Accuracy of the fitted models } \\
\text { (within sample) }\end{array}$} \\
\hline Mean Absolute \% error (MAPE) & \multicolumn{2}{|c|}{4.16} & \multicolumn{2}{|c|}{11.28} & \multicolumn{2}{|c|}{11.94} & \multicolumn{2}{|c|}{4.73} \\
\hline Mean Absolute Deviation (MAD) & \multicolumn{2}{|c|}{246.13} & \multicolumn{2}{|c|}{636.11} & \multicolumn{2}{|c|}{642.23} & \multicolumn{2}{|c|}{251.00} \\
\hline Mean Squared Deviation (MSD) & \multicolumn{2}{|c|}{95475.05} & \multicolumn{2}{|c|}{571104.90} & \multicolumn{2}{|c|}{572092.00} & \multicolumn{2}{|c|}{101231.10} \\
\hline Root Mean Sqaure Error (RMSE) & \multicolumn{2}{|c|}{308.99} & \multicolumn{2}{|c|}{755.71} & \multicolumn{2}{|c|}{756.37} & 318. & \\
\hline $\begin{array}{ll}\text { Relative forecast error (\%) } & \text { (Out } \\
\text { of sample, } 2001-2005) & \end{array}$ & 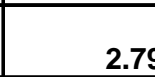 & & 23.2 & & & .52 & 5.9 & \\
\hline
\end{tabular}


Table 2: Accident prediction models for monthly car casualties within the congestion charging zone

\begin{tabular}{|c|c|c|c|c|c|c|c|c|}
\hline \multicolumn{9}{|c|}{ Disaggregate Time Series Accident Count Data (Monthly car casualties within the Congestion Charging Zone 1991 - 2004) } \\
\hline & \multicolumn{2}{|c|}{ SARIMA } & \multicolumn{2}{|c|}{ NB } & \multicolumn{2}{|c|}{ NB with a time trend } & \multicolumn{2}{|c|}{ INAR(1) Poisson } \\
\hline Explanatory Variables & Coeff & t-stat & Coeff & t-stat & Coeff & t-stat & Coeff & t-stat \\
\hline Congestion charge & -12.8624 & -2.85 & -0.3241 & -5.77 & -0.3193 & -5.46 & -0.3076 & -4.68 \\
\hline $\ln$ (Monthly Accidents) & 51.5318 & 3.19 & 0.7336 & 4.66 & 0.7179 & 4.32 & 0.8684 & 4.76 \\
\hline Time trend (Linear) & - & - & - & - & -0.0001 & -0.29 & - & - \\
\hline Constant & - & & -1.7157 & -1.35 & -1.5818 & -1.17 & -3.2373 & -2.18 \\
\hline Non-seasonal MA1 & 0.9361 & 24.22 & - & - & - & - & - & - \\
\hline Seasonal MA1 & 1.0646 & 11.90 & - & - & - & - & - & - \\
\hline Seasonal MA2 & -0.2127 & -2.52 & & & & & & \\
\hline \multicolumn{9}{|l|}{ Descriptive statistics } \\
\hline Overdispersion parameter & & & 0.0110 & 3.67 & 0.0110 & 3.67 & & \\
\hline Thinning parameter & & & & & & & 0.3545 & 10.46 \\
\hline Series of length & 168 & & 168 & & & & & \\
\hline Number of residuals & 155 & & 168 & & & & & \\
\hline \multicolumn{9}{|l|}{$\begin{array}{l}\text { Accuracy of the fitted models } \\
\text { (within sample) }\end{array}$} \\
\hline Mean Absolute \% error (MAPE) & \multicolumn{2}{|c|}{25.27} & \multicolumn{2}{|c|}{21.43} & \multicolumn{2}{|c|}{21.39} & \multicolumn{2}{|c|}{18.23} \\
\hline Mean Absolute Deviation (MAD) & \multicolumn{2}{|c|}{9.80} & \multicolumn{2}{|c|}{8.27} & \multicolumn{2}{|c|}{8.26} & \multicolumn{2}{|c|}{6.12} \\
\hline Mean Squared Deviation (MSD) & \multicolumn{2}{|c|}{146.82} & \multicolumn{2}{|c|}{104.63} & \multicolumn{2}{|c|}{104.12} & \multicolumn{2}{|c|}{95.36} \\
\hline Root Mean Sqaure Error (RMSE) & \multicolumn{2}{|c|}{150.36} & \multicolumn{2}{|c|}{126.93} & \multicolumn{2}{|c|}{126.63} & \multicolumn{2}{|c|}{105.23} \\
\hline $\begin{array}{l}\text { Relative forecast error (\%) (Out } \\
\text { of sample, Jan } 2005 \text { to Oct 2005) }\end{array}$ & \multicolumn{2}{|c|}{1.36} & \multicolumn{2}{|c|}{1.05} & \multicolumn{2}{|c|}{1.04} & \multicolumn{2}{|c|}{0.91} \\
\hline
\end{tabular}

